\title{
COMUNICAR LA POBLACIÓN: (DES)ARTICULACIONES EN EL DISCURSO PERIODÍSTICO EN MEDIOS DE PRENSA EN CUBA
}

\section{COMMUNICATE THE POPULATION: (DIS) ARTICULATIONS IN THE JOURNALISTIC DISCOURSE IN THE PRESS MEDIA IN CUBA}

\author{
Lisset Bourzac Macia ${ }^{1}$ \\ Iliana Benítez Jiménez ${ }^{2}$
}

Recibido: 2021-09-25 / Revisado: 2021-10-30 / Aceptado: 2021-11-25 / Publicado: 2022-01-01

Forma sugerida de citar: Bourzac-Macia, L. y lliana-Benítez, J. (2022). Comunicar la población: (des)articulaciones en el discurso periodístico en medios de prensa en Cuba. Retos de la Ciencia. 6(12). 41-50. https://doi.org/10.53877/rc.6.12.20220101.04

\section{RESUMEN}

El presente artículo se aproxima a la producción de noticias sobre dinámicas demográficas en medios de prensa del país, en el contexto de la COVID-19. Se realiza una revisión a las concepciones teóricas sobre la definición de comunicación en población, y los ejemplos que evidencian ciertas desarticulaciones en el discurso periodístico respecto a la temática. Convergen técnicas cualitativas como el análisis de contenido y la revisión bibliográfica y documental. El análisis se realiza a las versiones digitales de los periódicos Granma, Juventud Rebelde, Sierra Maestra, y al sitio Cubadebate, entre los meses de enero a mayo del año 2020. Se concluye que la Demografía en estrecho vínculo con la Comunicación, en tiempos de crisis sanitaria como la COVID-19, precisa la capacitación y superación constante de los profesionales del gremio en la interpretación y divulgación de problemas en torno a

\footnotetext{
1 Master en Estudios Cubanos y del Caribe. Profesora Instructora en la Universidad de Oriente. Cuba. E-mail: bourzatc@uo.edu.cu. / ORCID: http://orcid.org/0000-0002-5453-5807

2 Doctora en Ciencias Sociológicas. Profesora Titular en la Universidad de Oriente. Cuba. E-mail: ilianabj@uo.edu.cu / ORCID: http://orcid.org/0000-0002-4753-3416
} 
las dinámicas de población susceptibles a la reflexión y al cambio afín de garantizar un mejor dominio del tema y eliminar la repetitividad de las publicaciones.

Palabras clave: comunicación en población, discurso periodístico, COVID-19

\section{ABSTRACT}

This article approaches the production of news on demographic dynamics in the country's press media, in the context of COVID-19. A review is made of the theoretical conceptions about the definition of communication in the population, and the examples that show certain disarticulations in the journalistic discourse regarding the subject. Qualitative techniques such as content analysis and bibliographic and documentary review converge. The analysis is carried out on the digital versions of the Granma, Juventud Rebelde, Sierra Maestra newspapers, and on the Cubadebate site, between the months of January to May 2020. It is concluded that Demography is closely linked with Communication, in times of health crisis such as COVID-19, requires the training and constant improvement of the professionals of the union in the interpretation and dissemination of problems around population dynamics susceptible to reflection and change in order to guarantee a better mastery of the subject and eliminate the repeatability of the publications.

Key words: communication in population, journalistic discourse, COVID-19.

\section{INTRODUCCIÓN}

Los vínculos entre la Demografía y la Comunicación se han hecho cada vez más visibles en un intento por llevar al escenario público la representación de los principales desafíos de la sociedad contemporánea actual en cuanto a población y desarrollo. La Demografía, como ciencia de las proyecciones, tiene un fuerte componente cuantitativo, pues tiene como objeto el conteo de la humanidad a través de sus procesos vitales como nacimientos, muertes y migraciones, además de los factores que influyen en su comportamiento. La comunicación, como excelso arte de "poner en común", compartir, lograr consensos y, comunicar - en especial el periodismo con la función de noticiar (Haber, 2013, p.87)- tiene el encargo social de narrar, desde asentadas pautas cualitativas, todo lo que acontece en materia de población. ¿Cómo narrar lo que se cuenta? ¿Cómo cuenta lo narrado? Son retos inherentes al proceso de construcción de noticias. Preguntas, a su vez, que mueven la presente reflexión en torno al discurso periodístico sobre comunicación en población en medios de prensa cubanos.

El proceso de construcción de noticias en Cuba también da cuenta del necesario vínculo entre Demografía y Comunicación, aunque el camino transitado muestre aciertos y desaciertos al respecto. El presente artículo se aproxima a la producción de noticias sobre dinámicas demográficas en medios de prensa del país, en un intento por identificar algunas desarticulaciones en el discurso periodístico sobre comunicación en población.

Poner en contexto el tema a tratar implica una revisión a las concepciones teóricas realizadas sobre la definición de comunicación en población, y los ejemplos que evidencian ciertas desarticulaciones en el discurso periodístico respecto a la temática. Para ello convergen técnicas cualitativas como el análisis de contenido y la revisión bibliográfica y documental. El análisis se realiza a las versiones digitales de los periódicos Granma, Juventud Rebelde, Sierra Maestra, y al sitio Cubadebate, entre los meses de enero a mayo del año en curso. De la población total se 
seleccionan como muestra 50 trabajos periodísticos que trataron la temática demográfica, bajo el criterio de muestreo no probabilístico por conveniencia.

\section{DESARROLLO}

El tema demográfico y las dinámicas de las poblaciones de países desarrollados y en vías de desarrollo ha centrado los debates de la agenda de la organización de Naciones Unidas por varias décadas. Las Conferencias Mundiales de Población (CMP) han sido el espacio para ello, y han evidenciado la toma de conciencia de las naciones y los estados en relación con las transiciones demográficas y las variaciones de sus niveles de fecundidad, mortalidad y migraciones, al tiempo que expusieron la interrelación de las mismas con el desarrollo, la producción, la investigación, la educación y la comunicación.

A partir de 1954, en Roma comenzaron los debates sobre la población, con la mirada enfocada a los estudios en Demografía en países en desarrollo. Surgía así el interés por capacitar profesionales en asuntos de población y la intención de crear centros regionales para el análisis del comportamiento poblacional.

Una década después un mayor número de profesionales se dieron cita en Belgrado para debatir sobre la fecundidad como parte de una política de desarrollo, en el contexto de las estrategias controlistas y pronatalistas asumidas por los gobiernos para los cuales la superpoblación era entendida como un problema social. Sin embargo, en ambos cónclaves no se superó la perspectiva académica. La investigadora Dixie Edith Trinquete destaca de las "citas la posición positivista que redujo los debates y conclusiones a una evaluación básicamente estadística de las complejas dinámicas demográficas globales" (Trinquete, 2017: p. 5).

La conferencia de Bucarest en 1974, la primera de carácter intergubernamental, abrió paso a la comprensión de las dinámicas demográficas en su dimensión social y humana, en tanto planteó que el análisis no se trataba solo de cifras sino de sus repercusiones en las sociedades; mostró la interdependencia entre los factores de población y el desarrollo, y viabilizó la inclusión de políticas demográficas como parte de las políticas de avance social. Diez años después, en la cita de México se ratificaron los acuerdos tomados, afianzando el rol de la política en las dinámicas demográficas.

El cónclave celebrado en El Cairo, a pesar de no ser el más reciente marcó un hito en los análisis de población y desarrollo, y se mantiene como referente para la evaluación de los avances en este tema. A partir de él se incorporó el tema de población en las agendas políticas de los países de manera orgánica y coherente a través del Programa de Acción consensuado en la cita. Este programa destacó la urgencia de informar sobre los comportamientos demográficos y las decisiones asociadas a los mismos cuando plantea que:

"Es especialmente importante que las estrategias de información, educación y comunicación guarden relación con las políticas y estrategias nacionales de población y desarrollo y con toda una serie de servicios de salud reproductiva, incluidas la planificación de la familia y la salud sexual, y que los complementen, a fin de aumentar el uso de esos servicios y de mejorar la calidad del asesoramiento y de la atención. Los gobiernos, las organizaciones no gubernamentales y el sector privado deberían utilizar más y de forma más eficaz los programas de entretenimiento de los medios de difusión, como la radio y la televisión, el teatro popular y otros medios tradicionales" (Informe, 1994, p.2). 
Aparejado a la cita, en América Latina se desarrollaron experiencias que centraron los debates sobre temas demográficos desde los medios de prensa; de ahí el surgimiento de una comunicación en población, concepto que aún se enriquece con las nuevas demandas y las no tan nuevas prácticas periodísticas.

De los nexos entre los procesos demográficos y los medios de prensa nace la definición de comunicación en población. El concepto surgió en la década de 1990 del pasado siglo como resultado de la experiencia mexicana en la búsqueda de soluciones para enfrentar las problemáticas demográficas. Desde la fecha, la comunicación en población es la propuesta liderada por Rodolfo Tuirán que recoge las acciones para la educación y la comunicación sobre los procesos demográficos.

\section{Medios de comunicación y cultura demográfica. Los inicios de un debate.}

El inicio del debate en torno a la comunicación en población en Cuba se sitúa en la década de 1990. Desde el escenario académico, a través de una investigación sobre los niveles de mortalidad en el país (García, 1990; 1996) se vislumbraba la necesidad de abrir paso a otras ciencias en el diálogo constructivo para explicar las dinámicas demográficas de la sociedad cubana. Otras investigaciones (Rodríguez, 2006; Molina, 2017; Quintana, 2017) de marcado perfil demográfico denotaron la urgencia de incluir la perspectiva comunicológica en los análisis sobre las dinámicas de población. Ya fuese en temas vinculados a variables como la mortalidad y la fecundidad, o relacionadas con el envejecimiento y las peculiaridades de la Segunda Transición Demográfica en Cuba, los especialistas acentuaron la importancia de los procesos culturales, de la comunicación y de la influencia de la radio y la televisión en campañas de bien público orientadas a sensibilizar a la población en torno a sus principales retos demográficos.

Unido a los esfuerzos internacionales por incluir los procesos de educación, información y comunicación en las políticas de población, para la fecha en América Latina se gestaron experiencias de trabajo entre el Fondo de Población de Naciones Unida (UNFPA) y la agencia de noticias Inter PressService (IPS), las que dieron visibilidad a temáticas como el impacto demográfico del aborto, la esperanza de vida, la planificación familiar, las relaciones intergeneracionales, el envejecimiento de la población y las migraciones internas. Tres periodistas cubanos de medios oficiales contribuyeron a través de artículos y reportajes al tratamiento de la temática en periódicos y revistas a principios de la década de 1990. A razón de dos trabajos mensuales, cerca de casi tres años la población cubana conoció de las dinámicas poblacionales en dos medios nacionales: Juventud Rebelde, Bohemia y el semanario local Tribuna de La Habana. Aparejado a ello también hubo intenciones de superación profesional y capacitación en temas de población para periodistas a través del Centro Latinoamericano de Demografía (CELADE).

No obstante, la relación entre medios de comunicación y dinámicas demográficas aún era aislada en tanto las prácticas periodísticas eran mayormente visibles en medios nacionales. Asimismo, la presencia de los trabajos periodísticos tuvo un marcado carácter temporal que limitó la trascendencia de su contenido. La no mención a medios provinciales no asegura la ausencia del tratamiento periodístico a la temática, sino que manifiesta la regionalización que hasta la fecha han mostrado estos estudios.

En Cuba, ante el comportamiento de los niveles de fecundidad, mortalidad y migraciones surge la necesidad de la práctica de una comunicación en población que desde los medios de prensa permita articular un discurso en aras de alcanzar una cultura demográfica, y garantice en los ciudadanos los conocimientos y la 
participación consciente en sus dinámicas poblacionales. Al respecto, se enriquece el concepto con una propuesta teórica y científica concebida como:

"resultado de un proceso eminentemente social y se inscribe en un contexto en el que los retos que la población plantea han comenzado a hacerse socialmente visibles, más allá del dominio de la Demografía y la Comunicación, lo cual demanda de un enfoque transdisciplinar, entendiendo este como un proceso según el cual los límites de las disciplinas individuales se trascienden, para tratar problemas desde perspectivas múltiples con vista a generar conocimientos emergentes" (Trinquete, 2017: p. 47).

La propuesta teórica se fundamenta en las teorías comunicológicas que, a su vez, le aportan al binomio cultura-comunicación. La comunicación en población, por tanto, necesita del papel cada vez más activo de las audiencias, "generadoras de sentidos y productoras de contenidos". El término es pertinente en el análisis del reflejo de las dinámicas demográficas al mostrar que la relación población-desarrollo necesita tanto de las teorías difusionistas y de la interacción social asociadas a la transición demográfica, como del paradigma comunicativo centrado en los procesos de significación. Todo ello apunta hacia la elaboración de políticas integrales de población, por un lado, y el interés de estrategias de comunicación holísticas y coherentes, por otro.

(Des)articulaciones en los medios de prensa. El discurso periodístico sobre comunicación en población.

Las teorías de la Sociología del emisor y de la Mediación Social, fundamentalmente, han sido referentes en las investigaciones como la de Trinquete $(2011,2017)$ en torno a la construcción del discurso periodístico sobre comunicación en población. Las mismas han permitido identificar las principales mediaciones en el entramado del proceso de construcción de noticias basadas en las rutinas productivas e ideologías profesionales y su influencia en los discursos periodísticos sobre población.

Entre las desarticulaciones en el tratamiento periodístico a temas demográficos, connotan: la escasa presencia de la temática en medios nacionales; el predominio del género informativo; la carencia de competencias profesionales que permita la generación de contenido propio sobre el tema; la promoción institucional y académica por encima de la mediática sobre todo en coyunturas temporales específicas, la insuficiente preparación, capacitación y especialización periodística en determinados temas, y en ocasiones, el difícil acceso a los datos (Trinquete, 2011, Polanco, 2015, Bello, 2016 y Trinquete, 2017). Las mismas son resultado de mediaciones políticas, como la excesiva regulación de la política informativa y la ausencia de políticas integrales sobre Comunicación y sobre Población; y de mediaciones culturales como la gestión y organización de los flujos y rutinas productivas, además de la urgencia de una mirada integral a la cultura profesional del periodista y los directivos (Trinquete, 2017).

Una necesaria mirada en retrospectiva al proceso de construcción de noticias sobre comunicación en población, en particular desde el tratamiento periodístico a la fecundidad, destaca la presencia de tópicos de comunicación en población en la década de 1990 en el periódico provincial Sierra Maestra. Bourzac (2019) analiza los trabajos periodísticos relacionados con la temática y da cuenta de algunas de estas desarticulaciones en el discurso periodístico asociado a temas de población.

El tratamiento periodístico a la fecundidad en la década observada estuvo encaminado más a orientar a la población sobre Salud Sexual y Reproductiva (SSR) y la planificación familiar que, a explicar los determinantes próximos vinculados a esta 
variable demográfica, así como la influencia de los factores socio históricos y culturales en la dinámica de la fecundidad. En ello incidió la poca visualización del tema de la dinámica demográfica y su importancia para el desarrollo, por parte de la agenda política del país en esa década. Asimismo, se muestra la correspondencia con las prioridades de la agenda política, como la mortalidad infantil y los temas de familia, dejando de lado una valoración más profunda sobre la dinámica demográfica. Los análisis al respecto también evidenciaron predominio del empleo de género informativo, aún y cuando el espacio facilitaba otros formatos, y la recurrencia del uso del sector salud como principal fuente de información (Bourzac, 2019).

Comunicar la población es un reto para los medios de prensa y sus protagonistas en Cuba. En el estudio realizado a los medios de comunicación escogidos de enero a mayo del 2020 se observa que, dado el número de trabajos periodísticos sobre dinámicas de población, un total de 50 en los sitios digitales, el tema no constituye una prioridad en las agendas de los medios seleccionados. A pesar de la amplia cobertura de prensa a otras temáticas como salud, agricultura, economía, educación, deporte y cultura, no se acumula gran cantidad de trabajos periodísticos publicados en las versiones digitales de los periódicos Granma, Juventud Rebelde y Sierra Maestra, ni en la página oficial de Cubadebate. Diferencias significativas se observan en los diarios en relación con las características propias de estos, y los públicos a los que están dirigidos.

La producción de noticias relacionadas con la comunicación en población en el diario Juventud Rebelde en los meses observados se mantuvo con un promedio de entre 5 y 10 trabajos mensuales. En ello influye la variedad de secciones que muestra el rotativo, y en particular, una dedicada a la promoción de la educación sobre salud sexual y reproductiva dirigida por una periodista especializada en el tema, la sección Sexo Sentido. En el período escogido destaca la amplia cobertura de prensa a la situación sanitaria generada por la pandemia, como parte de la estrategia en el enfrentamiento a la misma. La avalancha informativa al respecto centró la agenda mediática en los meses de marzo, abril y mayo, situación que pudiera valer como justificación ante la ausencia de temas sobre comunicación en población. No obstante, se muestran publicaciones relacionadas con las dinámicas de población, aunque no presentan un análisis en profundidad.

Asimismo, resalta la publicación de trabajos relacionados con la salud sexual y reproductiva, ya sea desde un enfoque preventivo, o desde una mirada centrada en la orientación y educación a la población sobre las relaciones sexuales y la planificación familiar, como temas centrales, sobre todo en la sección Sexo Sentido de este mismo rotativo. De manera general, las publicaciones sobre la temática apenas representan el $2 \%$ de todo lo que publica en el diario cada mes. No obstante, predomina el comentario como la forma genérica para darle tratamiento al tema, en la que prevalece la opinión del periodista sin la correlación de otros criterios especializados. Más de un $50 \%$ de los trabajos analizados se asocian al sector de la salud como fuente principal de información. En la totalidad de los mismos se desestima el manejo de las estadísticas y por tanto muestran insuficiencias para una adecuada interpretación de las problemáticas.

Otro de los diarios cuya versión digital se seleccionó para el análisis del discurso periodístico sobre comunicación en población fue Granma. El sitio del Órgano Oficial del Partido Comunista de Cuba, publicó en el período, un total de 589 trabajos, de los cuales solo el 1,18\%, es decir, apenas 7 trabajos en cinco meses, fueron dedicados a informar sobre los retos del país en materia de atención a la dinámica demográfica. Todos centran la atención en la fecundidad como principal variable de atención, en 
particular con la mirada enfocada en la natalidad y las medidas para estimular el aumento de los nacimientos. De ellos, solo un trabajo publicado responde a los códigos del reportaje. El resto de las publicaciones se corresponde con el carácter marcadamente político del periódico en tanto muestra informaciones relacionadas con las medidas adoptadas, así como el seguimiento de la máxima dirección del país para el incremento de la natalidad.

Llama la atención, en este periódico, un trabajo periodístico sobre la intervención del subdirector de la Oficina Nacional de Estadísticas acerca la incidencia de la pandemia en la dinámica demográfica cubana. Como parte del texto la periodista afirma una idea que estriba en error, a veces frecuente, que se aleja de la situación real: "en el oriente del territorio nacional, donde la natalidad es mayor y, por tanto, la población joven es numéricamente superior, hay menos casos de la enfermedad en cuestión" (Silva, 17 de mayo de 2020).

En primer lugar, se está suponiendo que el aumento de la natalidad asegura directamente una población joven, lo que sucede sin el efecto de las migraciones y la mortalidad. Siempre que se hable del efecto de un hecho vital sobre la dinámica de la población se deberá analizar con relación al resto de las variables que inciden en ella, lo que requiere de una preparación en el análisis demográfico del periodista que trate el tema, al menos para que el entrevistado de explicación más precisa que permita hacerla llegar al público a la que va dirigida.

En segundo lugar, la existencia de una fecundidad ligeramente más elevada en las provincias orientales, que permita que la estructura por edades de la población en estas provincias sea más joven en relación con el resto, no implica que exista una menor incidencia de la enfermedad, pues ésta han mostrado diferenciales por provincias en Cuba que aún se encuentran en estudio y hasta el momento lo demostrado ha sido que la incidencia es mayor en las edades entre los 50 y 59 años, que no es la población de adultos mayores, mientras que la mayor mortalidad sí se hace mayor en las edades más avanzadas. En cualquier caso, se requiere de una fundamentación de las noticias que requiere de mayor dominio por parte del periodista de algunos elementos claves del análisis demográfico que le permita contrastar fuentes de información de manera crítica.

El periódico provincial Sierra Maestra, con un promedio de publicación mensual muy por debajo de los sitios anteriormente mencionados, aproximadamente de un 6 $\%$, registra un total de 14 trabajos vinculados directa o indirectamente con temáticas afines a las dinámicas de población. Se observa que el $75 \%$ de las publicaciones pertenecen al género información, y el resto responden a los códigos del comentario. Similar a lo que acontece en otros medios, el sector de salud es la principal fuente de información vinculada a los temas demográficos, en el caso particular del rotativo santiaguero, centrados en la salud sexual y reproductiva y con especial énfasis en el Programa de Atención Materno Infantil (PAMI). El discurso periodístico del semanario resalta como la variable demográfica más mencionada a la fecundidad y sus determinantes próximos: como la nupcialidad, la anticoncepción y el aborto; no obstante, se evidencia desarticulaciones en el tratamiento a la misma.

En la jornada del 27 de mayo la versión digital del Sierra Maestra publicó una información referida a los principales resultados del PAMI en la provincia con énfasis en la cantidad de nacimientos. Al respecto, se cita: "Entre 2018 y lo que va de 2020 el hospital acumula más de seis mil 500 nacimientos, lo cual contribuye a contrarrestar el envejecimiento poblacional que afecta a Cuba y, en particular, a la Ciudad Héroe" (Vaillant, 27 de mayo de 2020). Desde el enfoque periodístico pudiera parecer que la provincia con esa cifra por sí sola contribuye al aumento de la fecundidad y por ende, 
a una mayor cantidad de población joven en contraste con el alto grado de envejecimiento. Mas, la propia cita deja ver uno de los errores más comunes sobre el tema en nuestra prensa. Primeramente, las cifras absolutas como el total de nacimientos no dejan ver si realmente la proporción de los mismos con respecto al tamaño de la población ha sido realmente mayor, por lo que es incorrecto su uso.

También es errada la concepción que se maneja de envejecimiento poblacional, como una afectación que tiene Cuba. Similar a lo comentado anteriormente, es necesario comprender qué significa un envejecimiento demográfico o de la estructura por edades de la población. No se trata de una correspondencia proporcional entre el envejecimiento como consecuencia del comportamiento solo de la baja fecundidad, sino que debe analizarse la intervención del resto de las variables demográficas como la mortalidad y las migraciones. Este refleja el incremento de la proporción de personas de 60 años y más, respecto al total de la población. Por tanto, se llega a este estado por la disminución de la fecundidad, pero también por la disminución de las tasas de mortalidad y extensión la esperanza de vida de la población cubana, lo cual es un logro, en especial del sistema de salud cubano y las políticas sociales que apoyan tal dinámica. El envejecimiento demográfico precisa de una atención diferenciado por los retos que impone, pero no es un problema en sí. Igualmente queda pendiente de mayor atención la emigración de población en edades jóvenes, que constituyen los recursos laborales necesarios para garantizar la producción de bienes en esa población que asegure su desarrollo.

Lo anterior, por demás bastante frecuente en las publicaciones cubanas, es expresión de la carencia de capacitaciones y actualizaciones sobre el tema brindadas a los periodistas, y de lo engorroso que resulta la temática. De igual manera, evidencia la ausencia del contraste de fuentes y la poca profundidad en el análisis de lo expuesto.

Mientras, el sitio digital Cubadebate se posiciona como uno de los mejores exponentes en la producción de noticias sobre comunicación en población en el período analizado. El tratamiento periodístico a las dinámicas de población forma parte del contenido de la sección Letras de género, publicada en el sitio desde enero de 2020, con una frecuencia semanal. Las periodistas Dixie Edith Trinquete y Ania Terrero, comparten extensos reportajes relacionados con la temática de la igualdad de género, derechos y salud sexual y reproductiva en mujeres y hombres, maternidad y paternidad responsables, calidad de vida y embarazos, así como garantías para mujeres y niños en Cuba. En todos destaca el manejo de las estadísticas sobre la situación demográfica cubana con un análisis en profundidad, con un contraste de las fuentes, en función, sobre todo, de la reflexión que emerge tras el examen riguroso a los datos oficiales.

De esta manera, la práctica periodística en el sitio Cubadebate con relación a los temas de población evidencia cómo debe articularse la construcción de noticias que refleje oportuna, profunda y acertadamente los principales desafíos en materia demográfica, haciendo uso de la diversidad genérica, la variedad de las fuentes, la investigación periodística y el estilo propio de cada uno de los redactores.

\section{CONCLUSIONES}

La construcción de noticias sobre comunicación en población aún muestra desarticulaciones en el discurso periodístico en medios de prensa del país. No se trata de un problema de espacio, como en los formatos impresos de cada uno de los diarios y semanarios; sino una cuestión de interés, un asunto que ha de volverse 
prioridad para los periodistas y a tono con la agenda, en correspondencia con las características del medio de comunicación. La Demografía en estrecho vínculo con la Comunicación, precisa la capacitación y superación constante de los profesionales del gremio en la interpretación y divulgación de problemas en torno a las dinámicas de población susceptibles a la reflexión y al cambio afín de garantizar un mejor dominio del tema y eliminar la repetitividad de las publicaciones. Asimismo, es válido ampliar el diapasón temático y no centrarse solo en el tratamiento a la variable fecundidad y sus determinantes próximos, y en menor medida el envejecimiento y la mortalidad; sino que es válido pensar también en la interrelación de estas con las migraciones, y en particular en el comportamiento propio de este indicador demográfico, ausente de toda mención en la prensa.

Es sin dudas, un gran reto para el periodismo interpretativo y de datos, un camino aún por transitar en nuestras redacciones. Empero, no se aboga por una especialización, en tanto se precisa comprender que "lo demográfico" en la prensa cubana hoy es transversal a todos los asuntos de interés. Se trata en primer lugar, de desterrar los obstáculos que a veces imponen el manejo de los datos, tanto en su análisis como en su obtención; dejar de lado el facilismo en la producción de noticias y explorar aristas, no tan nuevas, pero sí interesantes. Comunicar los desafíos poblacionales deviene en pretexto para explotar los géneros de opinión, agudizar la mirada en aquellos elementos que las cifras por sí solas no dejan ver y emplear una diversidad de fuentes que den la posibilidad de un análisis más profundo y crítico. Se trata de entender que una buena práctica de comunicación en población con el fin de generar conocimientos emergentes, desde la prensa, contribuya a dar al traste con la visión errada de problemas de población para viabilizar respuestas y soluciones a las poblaciones con problemas.

\section{REFERENCIAS BIBLIOGRÁFICAS}

Benítez, I. (2013): Estudio sociológico de la relación entre el comportamiento reproductivo, la dinámica poblacional y el desarrollo social en el municipio Santiago de Cuba. (Tesis doctoral) Universidad de Oriente.

Bello, E. (2016) Comunicación en población en los medios de comunicación de Holguín. (Tesis de Maestría) Universidad de La Habana.

Bourzac, L. (2019): Nacer en los 90. Tratamiento periodístico a la fecundidad en el periódico Sierra Maestra. (Tesis de Maestría) Universidad de Oriente.

García, R. (1990) Primer Taller sobre prioridades de investigación en materia de mortalidad en Cuba. Centro de Estudios Demográficos de la Universidad de La Habana.

(1996). La transición de la mortalidad en Cuba. Un estudio sociodemográfico. Centro de Estudios Demográficos.

Haber, Y. (2013): Tinta negra para recursar el periodismo. Oriente.

Molina, M. (2017). La fecundidad adolescente en Cuba. CEDEM.

Naciones Unidas (1994). Conferencia Internacional sobre Población y Desarrollo El Cairo. https://bit.ly//unfpa.org/.

Polanco, C. (2015). Comunicación y Demografía: vínculo necesario. (Tesis de Licenciatura en Periodismo). Universidad de La Habana.

Quintana, L. (2017). Cuba: Fecundidad y toma de decisiones en torno a la reproducción. Miradas en contexto. (Tesis Doctoral) Universidad de La Habana. 
Rodríguez, G. (2006). La fecundidad cubana a partir de 1990. Las perspectivas sociales e individual. (Tesis doctora). Universidad de La Habana.

Silva, Y. (2020, may 17) El envejecimiento de la población, un reto en el enfrentamiento a la pandemia. Granma. http://bit.ly//granma.cu/.

Trinquete, D. (2011) Mediaciones para el tratamiento de la fecundidad en la prensa escrita cubana. (Tesis de Maestría) Universidad de La Habana.

(2015): La Comunicación en Población en el contexto de los problemas sociales de la ciencia y la tecnología. Novedades en Población, 10(20), 105115. La Habana.

(2017). Contar la sociedad de la demografía a la comunicación. (Tesis doctoral) Universidad de La Habana.

Tuirán, R. (1996): Cultura demográfica. Comunicación en población y procesos de difusión. Revista DemoS, No. 9 Pp 25-26. UNAM.

Vaillant, B (2020, may 27) Exhibe hospital santiaguero positivos indicadores del PAMI en Sierra Maestra. http://bit.ly//sierramaestra.cu/. 